\title{
Effect of a Multicomponent Behavioral Intervention in Adults Impaired by Psychological Distress in a Conflict-Affected Area of Pakistan A Randomized Clinical Trial
}

Atif Rahman, PhD; Syed Usman Hamdani, MBBS; Naila Riaz Awan, PhD; Richard A. Bryant, PhD; Katie S. Dawson, PhD; Muhammad Firaz Khan, MRCPsych; Mian Mukhtar-ul-Haq Azeemi, MBBS; Parveen Akhtar, MPhil; Huma Nazir, BS (Hons); Anna Chiumento, MSc; Marit Sijbrandij, PhD; Duolao Wang, PhD; Saeed Farooq, PhD; Mark van Ommeren, PhD

IMPORTANCE The mental health consequences of conflict and violence are wide-ranging and pervasive. Scalable interventions to address a range of mental health problems are needed.

OBJECTIVE To test the effectiveness of a multicomponent behavioral intervention delivered by lay health workers to adults with psychological distress in primary care settings.

DESIGN, SETTING, AND PARTICIPANTS A randomized clinical trial was conducted from November 1, 2014, through January 28, 2016, in 3 primary care centers in Peshawar, Pakistan, that included 346 adult primary care attendees with high levels of both psychological distress and functional impairment according to the 12-item General Health Questionnaire and the World Health Organization Disability Assessment Schedule 2.0 (WHODAS 2.0).

INTERVENTIONS Lay health workers administered 5 weekly 90 -minute individual sessions that included empirically supported strategies of problem solving, behavioral activation, strengthening social support, and stress management. The control was enhanced usual care.

MAIN OUTCOMES AND MEASURES Primary outcomes, anxiety and depression symptoms, were independently measured at 3 months with the Hospital Anxiety and Depression Scale (HADS). Secondary outcomes were posttraumatic stress symptoms (Posttraumatic Stress Disorder Checklist for DSM-5), functional impairment (WHODAS 2.0), progress on problems for which the person sought help (Psychological Outcome Profiles), and symptoms of depressive disorder (9-item Patient Health Questionnaire).

RESULTS Among 346 patients (mean [SD] age, 33.0 [11.8] years; $78.9 \%$ women), 172 were randomly assigned to the intervention and 174 to enhanced usual care; among them, 146 and 160 completed the study, respectively. At baseline, the intervention and control groups had similar mean (SD) HADS scores on symptoms of anxiety (14.16 [3.17] vs 13.64 [3.20]; adjusted mean difference [AMD], 0.52; 95\% Cl, -0.22 to 1.27) and depression (12.67 [3.27] vs 12.49 [3.34]; AMD, $0.17,95 \% \mathrm{Cl},-0.54$ to 0.89 ). After 3 months of treatment, the intervention group had significantly lower mean (SD) HADS scores than the control group for anxiety (7.25 [3.63] vs 10.03 [3.87]; AMD, $-2.77 ; 95 \% \mathrm{Cl},-3.56$ to -1.98 ) and depression (6.30 [3.40] vs 9.27 [3.56]; AMD, $-2.98 ; 95 \% \mathrm{Cl},-3.74$ to -2.22$)$. At 3 months, there were also significant differences in scores of posttraumatic stress (AMD, $-5.86 ; 95 \% \mathrm{Cl},-8.53$ to -3.19 ), functional impairment (AMD, -4.17; $95 \% \mathrm{Cl},-5.84$ to -2.51 ), problems for which the person sought help (AMD, $-1.58 ; 95 \% \mathrm{Cl},-2.40$ to -0.77 ), and symptoms of depressive disorder (AMD, $-3.41 ; 95 \% \mathrm{Cl},-4.49$ to -2.34 ).

CONCLUSIONS AND RELEVANCE Among adults impaired by psychological distress in a conflict-affected area, lay health worker administration of a brief multicomponent intervention based on established behavioral strategies, compared with enhanced usual care, resulted in clinically significant reductions in anxiety and depressive symptoms at 3 months.

TRIAL REGISTRATION anzctr.org.au Identifier: ACTRN12614001235695

JAMA. doi:10.1001/jama.2016.17165

Published online November 12, 2016.
Supplemental content
Author Affiliations: University of Liverpool, Liverpool, England (Rahman, Chiumento); Human Development Research Foundation, Islamabad, Pakistan (Hamdani, Akhtar, Nazir); Lady Reading Hospital, Peshawar, Pakistan (Awan, Khan, Azeemi, Farooq); University of New South Wales, Sydney, Australia (Bryant, Dawson); VU University Amsterdam, Amsterdam, the Netherlands (Sijbrandij); Liverpool School of Tropical Medicine, Liverpool, England (Wang); Research Institute for Primary Care \& Health Sciences, Keele University, Staffordshire, England (Farooq); World Health Organization, Geneva, Switzerland (van Ommeren). Corresponding Author: Atif Rahman, PhD, Institute of Psychology, Health, and Society, University of Liverpool, 1-5 Dover St, Block B, Waterhouse Bldg, Liverpool L69 3BX, England (atif.rahman@liverpool.ac.uk). 
$\mathrm{M}$ ore than 125 million people today are directly affected by armed conflict, the highest number since World War II. ${ }^{1}$ Although reported rates of mental disorders vary, a meta-analysis of a subset of relatively rigorous postconflict surveys showed that mood and anxiety disorders were common, with rates of $17.3 \%$ for depression and $15.4 \%$ for posttraumatic stress disorder. ${ }^{2}$

Psychological interventions are among the first-line treatments for mood, anxiety, and posttraumatic stress disorders. ${ }^{3,4}$ A key barrier to sustainable delivery of mental health care in low-income countries, particularly in areas affected by conflict or disaster, is the scarcity of human resources. ${ }^{5}$ The implementation of adapted psychological interventions by supervised lay health workers is a potential solution receiving significant attention as part of the global mental health research agenda. ${ }^{6,7}$

The World Health Organization (WHO), as part of its Mental Health Gap Action Programme, has begun to release a series of manualized psychological interventions adapted for scaled-up delivery by lay health workers. A brief psychological intervention incorporating behavioral strategies was developed as part of this series. ${ }^{8}$ In a previous study, the intervention was adapted for use in primary care in Pakistan, using established methods we have used previously. ${ }^{9} \mathrm{~A}$ small pilot trial in the conflict-affected city of Peshawar, Pakistan, showed that the intervention was feasible, integrated well into the primary care system, and improved functioning and posttraumatic stress symptoms compared with enhanced care as usual. ${ }^{10}$

The aim of the present study was to test the intervention through a larger randomized clinical trial (RCT). We hypothesized that primary care attendees randomly assigned to the intervention would show greater reductions in symptoms of anxiety, depression, posttraumatic stress, and functional impairment and reduced symptoms of depressive disorder after treatment and at 3-month follow-up compared with those randomized to enhanced usual care.

\section{Methods}

Design

The study was a single-blind individual RCT conducted from November 1, 2014, through January 28, 2016 (Figure). Primary outcomes were symptoms of anxiety and depression 3 months after treatment began. Secondary outcomes were symptoms of posttraumatic stress, functional impairment, problems for which the person sought help, and rates of symptoms of depressive disorder. The project was approved locally by the Institutional Review and Ethics Board of the Postgraduate Medical Institute, Lady Reading Hospital, Peshawar, and by the WHO Ethical Review Committee. All participants provided written informed consent. The full trial protocol ${ }^{11}$ is available in Supplement 1.

Participants

Participants were routine patients from 3 primary care centers in Peshawar, Pakistan, aged between 18 and 60 years.

\section{Key Points}

Question What is the effectiveness of a brief multicomponent intervention incorporating behavioral strategies delivered by lay health workers to adults functionally impaired by symptoms of psychological distress in a conflict-affected setting?

Findings In a randomized clinical trial in primary care settings in Peshawar, Pakistan, 346 adults impaired by psychological distress were randomized to the intervention or enhanced usua care. After 3 months of treatment, the intervention group had significantly lower anxiety and depression scores and lower rates of depressive disorder compared with those in the enhanced usual care group.

Meaning This lay worker-administered intervention may be a practical approach for treating adults with psychological distress in conflict-affected areas.

The primary care centers, covering a population of 30000 to 50000 individuals each, are government facilities staffed by a physician, midwife, paramedic staff, and community health workers providing general health care and maternity services. All participants clinically assessed by their physician to be currently experiencing emotional distress were invited to participate in the study. The physicians were trained to recognize common mental disorders. Demographic data obtained for this study included age, sex, education, and experience of stressful events in the last year.

\section{Procedures}

Invited participants were approached by a trained research assistant to obtain written informed consent to screen for trial eligibility. Consenting participants were then screened by trained research assistants; those who scored both 3 or higher on a screening questionnaire for common mental disorders (12-item General Health Questionnaire [GHQ-12] $)^{12,13}$ and 17 or higher on a questionnaire for functional impairments (WHO Disability Assessment Schedule 2.0 [WHODAS 2.0] $)^{14}$ were invited to participate. The GHQ-12 has 12 questions scored on a 4-point Likert scale. When used as screener, it is scored bimodally (ie, 0-0-1-1) with a total score ranging from 0 to 12 , where higher scores indicate higher likelihood of morbidity. A cutoff of 3 or higher can be used to indicate clinical caseness of common mental disorders in Pakistan. ${ }^{13}$ The WHODAS 2.0 is further described in the "Secondary Outcomes" section. Exclusion criteria were imminent risk of suicide, severe mental disorder (eg, psychotic disorders, substance dependence), or severe cognitive impairment (eg, severe intellectual disability, dementia).

Eligible participants were invited to take part in the RCT. All assessments were performed by trained research assistants blind to allocation status of participants who received 2-day training covering administration of instruments, general interview techniques, and ethical research conduct, in addition to a half day of security training.

Randomization was conducted after baseline assessment by an independent research assistant located off site 
and not involved in any other aspect of the study. Randomization was performed using computerized software on 1:1 basis. Allocation concealment was ensured by keeping the random assignments in sequentially numbered, opaque, sealed envelopes at the off-site center. The trial coordinator contacted each participant after being informed of participants' allocation status. Participants randomized to the intervention group were allocated a trained lay health worker by the trial coordinator. This health worker contacted the participant to plan the 5 consecutive sessions with them, with the first session scheduled no longer than 1 week after the pretreatment assessment. Posttreatment assessments were carried out 1 week after treatment and 3 months following the start of treatment. Participants in both groups who developed severe psychiatric problems (eg, psychosis, imminent suicidality) that required immediate specialist treatment and follow-up were referred to the Lady Reading Hospital for specialist care.

\section{Intervention}

The brief multicomponent intervention, called Problem Management Plus (PM+), was based on established problemsolving and behavioral techniques. ${ }^{8,15}$ The intervention was transdiagnostic (ie, targeted symptoms across a range of conditions rather than being diagnosis specific). It consisted of 5 weekly face-to-face sessions lasting 90 minutes each. ${ }^{15}$ Session 1 oriented participants to the intervention with motivational interviewing techniques to improve engagement, provided information about common reactions to adversity, and taught participants a basic stress management strategy (slow breathing). This strategy was practiced at the conclusion of every session to enhance its learning. Session 2 addressed a participant-selected problem using problem-solving techniques and introduced behavioral activation during which individuals were encouraged to reengage gradually with pleasant and task-oriented activities to improve mood and functionality. Sessions 3 and 4 supported participants' continued application of problem solving, behavioral activation, and stress management and introduced strategies to strengthen social support networks. In session 5, education about retaining treatment gains was provided and all learned strategies were reviewed.

The intervention is available on the WHO website ${ }^{15}$ and in the eAppendix in Supplement 2.

The training and supervision model for lay health workers followed on-the-job training. ${ }^{16}$ In this study, the lay health workers had 12 to 16 years of education with no previous clinical training or experience in counseling, social work, clinical psychology, or psychiatry. The master trainer (K.S.D.) conducted a 6-day training with local mental health specialists who in turn provided an 8-day training program to 9 lay health workers. Both training programs comprised education about common mental disorders, basic counseling skills, delivery of intervention strategies, and self-care. Supervisors received additional training in training and supervision skills. Training of both supervisors and health workers was followed by clinical practice with 3 practice cases each under supervision. Following
Figure. Flow Diagram of Progress Through Phases of a Randomized Trial Comparing a Brief Psychological Intervention vs Enhanced Usual Care Among Primary Care Attendees in Conflict-Affected Pakistan

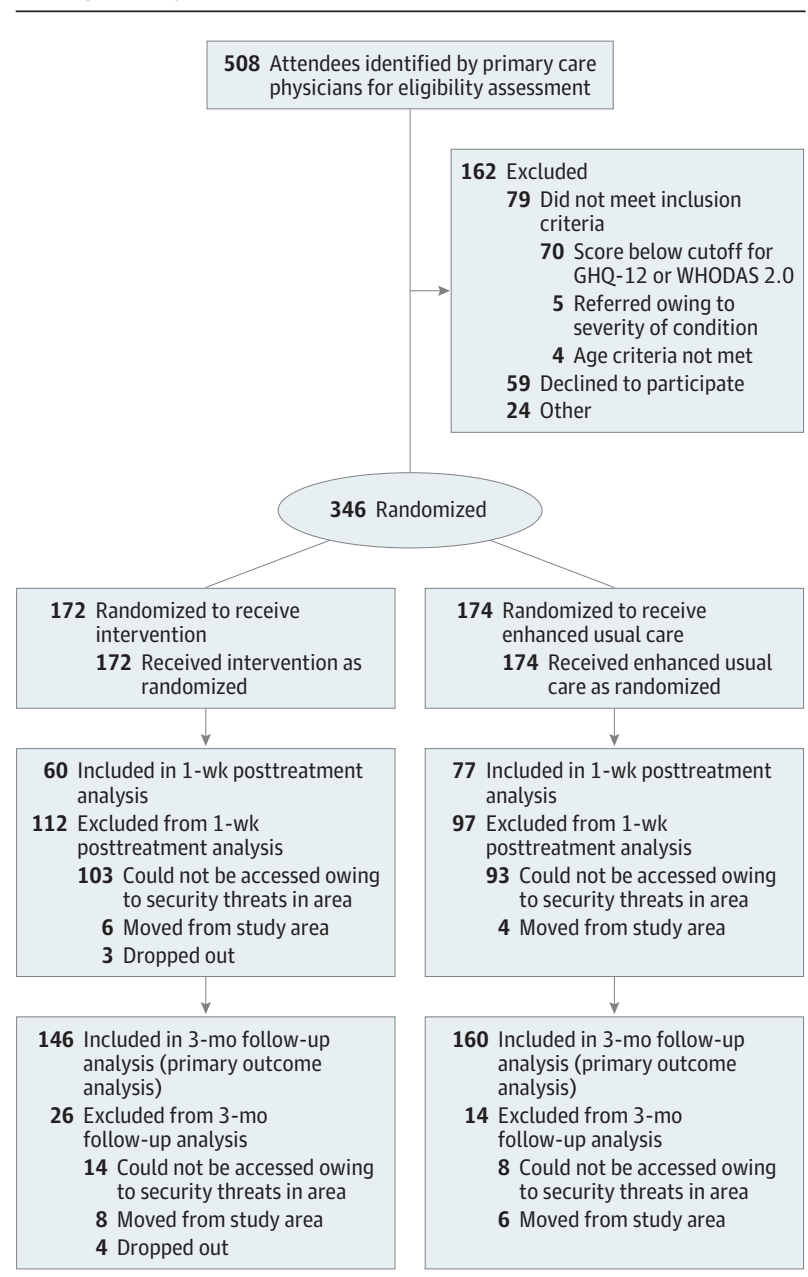

GHQ-12 indicates 12-item General Health Questionnaire; WHODAS 2.0, World Health Organization Disability Assessment Schedule 2.0.

this, all lay health workers were assessed for their competency in delivering the intervention using a competency rating tool that evaluated basic counseling skills and for their use of intervention strategies with clients through direct observation of specially designed role plays. Competency was rated using a 5-point Likert scale ranging from 0 (not done) to 5 (excellent). A score of 2 or higher on each item indicated competency.

The lay health workers were supervised in 2 groups on a weekly basis ( 2 hours) by the in-country supervisors. The latter were supervised (1-2 hours per month by Skype) by the master trainer, building their skills in the intervention and in training and supervision of others. One in-country supervisor (N.R.A.) directly observed a randomly selected sample of $10 \%$ of health workers' sessions ( $n=80 ; 10$ sessions per health worker) and used a checklist to systematically assess fidelity to the intervention. The session-wise checklist consisted of items capturing key intervention strategies for each session. The responses were recorded as yes or no 
for each given strategy for the particular session. Based on this evaluation, the supervisor rated each session overall as satisfactory or unsatisfactory in terms of fidelity achieved. Identified weak areas were reinforced during supervision.

\section{Enhanced Usual Care}

Primary care physicians in the participating centers received a 5-day training course run by the Lady Reading Hospital as part of the national community mental health program. ${ }^{17}$ This was enhanced through 1-day refresher training on common presentations of anxiety and depression, psychoeducation, supportive counseling, rational use of antidepressants or antianxiety medication, and referral pathways. Participants randomized to enhanced usual care were seen at least once by their primary care physician. Study participants and their accompanying family member were provided psychoeducation and the opportunity to talk about their health in a supportive environment. Participants were given the option of a repeated consultation.

\section{Primary Outcomes}

The primary outcomes were severity of anxiety and depressive symptoms measured independently using the Hospital Anxiety and Depression Scale (HADS). ${ }^{18,19}$ The HADS is a wellestablished 14-item scale consisting of 2 subscales: HADS-A (anxiety; 7 items; possible score range, 0-21) and HADS-D (depression; 7 items; possible score range, 0-21). Higher scores indicate more anxiety and/or depression. The HADS has been widely used across cultures, ${ }^{20}$ including Pakistan, ${ }^{19}$ and found to have good reliability and validity. The minimal clinically important difference has been determined at 1.32 for HADS-A and 1.40 for HADS-D. ${ }^{21}$

\section{Secondary Outcomes}

Posttraumatic stress disorder symptoms were measured using the 20-item Posttraumatic Stress Disorder Checklist for DSM-5 (PCL-5). ${ }^{22}$ Items are rated on a scale of 0 to 4 with a total score range of 0 to 80 . The PCL- 5 has shown good psychometric properties in terms of diagnostic accuracy and internal consistency ${ }^{23}$ and has been used in Pakistan. ${ }^{24}$ To improve sensitivity to change, the PCL- 5 was adapted by asking for symptoms in the past week rather than month.

Functional impairment was measured by the WHODAS $2.0,{ }^{14}$ which assesses health-related difficulties across domains of functioning. Difficulties are scored on a 5-point scale over the past 30 days. The 12-item interviewer-administered version was used, which is applicable across all health states including mental disorders. The WHODAS 2.0 has shown good validity in terms of internal consistency, test-retest reliability, and agreement with other measures of disability across countries. ${ }^{25}$

The Psychological Outcome Profiles (PSYCHLOPS) ${ }^{26}$ instrument was used to measure progress on problems for which the person sought help. It covers 3 domains: problems (2 questions), functioning (1 question), and well-being (1 question). Responses are scored on an ordinal 6-point scale, with a maximum score of 20 (5 points per question). The PSYCHLOPS has shown satisfactory internal consistency, good convergent va- lidity with measures of psychological distress, and high sensitivity to change. ${ }^{27}$

Participants were assessed for symptoms of depressive disorder with the 9-item Patient Health Questionnaire (PHQ-9), an instrument that incorporates DSM-IV depression diagnostic criteria with other key major depressive symptoms. ${ }^{28}$ Participants rate their responses on a 4-point Likert scale ranging from not at all to nearly every day. The PHQ-9 total severity score ranges from 0 to 27. The PHQ-9 has been validated in the Urdu language, showing adequate sensitivity and specificity. ${ }^{29,30}$ A cutoff score of 10 or higher was used to diagnose depressive disorder.

A modified version of the Life Events Checklist previously developed for the Pakistani population ${ }^{31}$ was used to identify stressful life events in the previous year rated as either present or absent. Data on life events were collected at 3 months' follow-up only.

\section{Statistical Analysis}

A total of 346 participants were included in this study. Because no comparable intervention studies in this population have been carried out, we aimed for a relatively conservative estimate of a 50\% reduction in HADS overall score in the intervention group as compared with a $30 \%$ reduction in the control group at 3 months' follow-up (this estimate was extrapolated from a trial involving lay workers in India) ${ }^{32}$ with a mean (SD) HADS score of 25 (8) at baseline. This corresponded with an effect size of 0.625 in the total HADS score. We assumed that the effect sizes for the HADS-A and HADS-D subscales would be similar to that for the total HADS score. Power calculations suggested a minimum sample size of 133 participants per group (power $=0.95$; $a=.05,2$-sided). To account for a conservative $30 \%$ attrition at 3 months' follow-up, we aimed to include a total of 346 participants (173 in the intervention group and 173 in the enhanced usual care group).

The primary focus of analyses was on intent-to-treat analyses consisting of all patients included according to the groups in which they were randomized. Using SPSS version 23 (SPSS Inc) and SAS version 9.3 (SAS Institute Inc) statistical software, we used linear mixed models to study treatment effects. This allows the number of observations to vary between participants and effectively handles missing data. ${ }^{33}$ The mixed model uses a longitudinal data structure that includes both fixed and random effects. Time (linear and quadratic), treatment, and interactions between treatment and time (linear and quadratic) were included as fixed effects in the mixed models and participant was included as random effect. Differences in the least squares means (treatment effects) at each time point with $95 \%$ confidence intervals were derived. A 2 -sided $P<.05$ was used to define statistical significance. Fixed-effects parameters were tested with the Wald test.

Primary analyses focused on HADS-A and HADS-D scores. Secondary analyses focused on results from the PCL-5, WHODAS 2.0, PSYCHLOPS, and PHQ-9. In a post hoc analysis, changes in participants meeting cutoff scores for depressive disorder were calculated for the treatment completer 
sample using the recommended cutoff of 10 on the PHQ- $9^{28}$ and analyzed using a linear mixed model with the same fixed and random effects as mixed models described earlier, from which the risk difference and 95\% confidence interval at each time point were derived. Another post hoc analysis of primary and secondary outcomes was conducted in which the baseline measurement of an outcome was treated as a covariate. Finally, post hoc sensitivity analysis using multiple imputation was performed to assess the robustness of treatment effect to the missing values. None of the post hoc analyses were prespecified in the protocol.

\section{Results}

Competency assessments following training found that 8 of 9 health workers scored 2 or higher on all items of the basic counseling skills and intervention strategies assessed. Of the 80 sessions observed directly to evaluate fidelity, 68 (85.0\%) achieved satisfactory fidelity to the intervention.

The Figure depicts participant flow through the trial. At the primary outcome assessment point of 3 months following the start of treatment, follow-up was available for 146 of 172 participants in the intervention group (84.9\%) and 160 of 174 participants in the control group (92.0\%). At 1 week after treatment, the follow-up rates were $34.9 \%$ in the intervention group and $44.3 \%$ in the control group owing to security threats at the time.

The mean (SD) number of sessions attended by the intervention group was 4.2 (1.70) and the duration of each session was about 90 minutes. Data were not available on the number or duration of sessions with the primary care physician in the control group. No participants were prescribed psychotropic medication by the primary care centers for the duration of the study.

Table 1 provides information on demographic characteristics, exposure to life events, and traumatic events for the sample. Of the 346 total participants, 273 (78.9\%) were female, 203 (58.7\%) were uneducated, 210 (60.7\%) had witnessed or experienced armed conflict or war, 91 (26.3\%) had witnessed or experienced physical assault, and 70 (20.2\%) had witnessed or experienced natural disaster. The mean (SD) age of the participants was 33.0 (11.8) years. There were no differences between groups in demographic characteristics except in education, with more participants in the control group having no education. The majority of participants in both groups were women. According to the PSYCHLOPS, the top 3 self-identified problems were headache (124 participants [35.8\%]); fatigue, lethargy, or low energy (99 participants [28.6\%]); and sleep problems (46 participants [13.3\%]).

Table 2 presents the findings for the primary outcomes of anxiety (HADS-A) and depression (HADS-D) and the secondary outcomes of posttraumatic stress (PCL-5), functional impairment (WHODAS 2.0), problems for which the person sought help (PSYCHLOPS), and symptoms of depressive disorder (PHQ-9) in the intervention and control groups at all time points. At baseline, the intervention and control groups had similar scores on symptoms of anxiety (mean

\begin{tabular}{|c|c|c|c|}
\hline \multirow[b]{3}{*}{ Variable } & \multicolumn{3}{|l|}{ №. (\%) } \\
\hline & \multirow[b]{2}{*}{$\begin{array}{l}\text { Total } \\
(\mathrm{N}=346)\end{array}$} & \multicolumn{2}{|l|}{ Group } \\
\hline & & $\begin{array}{l}\text { Intervention } \\
(\mathrm{n}=172)\end{array}$ & $\begin{array}{l}\text { Enhanced } \\
\text { Usual Care } \\
(\mathrm{n}=174)\end{array}$ \\
\hline Age, mean (SD), y & $33.0(11.8)$ & $32.7(12.1)$ & $33.4(11.4)$ \\
\hline \multicolumn{4}{|l|}{ Sex } \\
\hline Male & $73(21.1)$ & $43(25.0)$ & $30(17.2)$ \\
\hline Female & $273(78.9)$ & $129(75.0)$ & $144(82.8)$ \\
\hline \multicolumn{4}{|l|}{ Education } \\
\hline Uneducated & $203(58.7)$ & $91(52.9)$ & $112(64.4)$ \\
\hline \multicolumn{4}{|l|}{ Completed } \\
\hline Primary, grade 5 & $42(12.1)$ & $22(12.8)$ & $20(11.5)$ \\
\hline Middle, grade 8 & $26(7.5)$ & $16(9.3)$ & $10(5.7)$ \\
\hline $\begin{array}{l}\text { Matriculate, } \\
\text { grade } 10\end{array}$ & $29(8.4)$ & $18(10.5)$ & $11(6.3)$ \\
\hline $\begin{array}{l}\text { College and } \\
\text { university, } \\
\text { grades 11-16 }\end{array}$ & $46(13.3)$ & $25(14.5)$ & $21(12.1)$ \\
\hline \multicolumn{4}{|l|}{$\begin{array}{l}\text { Stressful events } \\
\text { in past year }\end{array}$} \\
\hline \multicolumn{4}{|l|}{$\begin{array}{l}\text { Witnessed } \\
\text { or experienced }\end{array}$} \\
\hline $\begin{array}{l}\text { Armed conflic } \\
\text { t or war }\end{array}$ & $210(60.7)$ & $107(62.2)$ & $103(59.2)$ \\
\hline Natural disaster & $70(20.2)$ & $32(18.6)$ & $38(21.8)$ \\
\hline Serious road accident & $180(52.0)$ & $91(52.9)$ & $89(51.1)$ \\
\hline Physical assault & $91(26.3)$ & $42(24.4)$ & $49(28.2)$ \\
\hline $\begin{array}{l}\text { Unnatural death } \\
\text { of family or friend }\end{array}$ & $38(11.0)$ & $16(9.3)$ & $22(12.6)$ \\
\hline Serious injury to self & $26(7.5)$ & $14(8.1)$ & $12(6.9)$ \\
\hline $\begin{array}{l}\text { Ill health with no access } \\
\text { to medical care }\end{array}$ & $21(6.1)$ & $10(5.8)$ & $11(6.3)$ \\
\hline
\end{tabular}

[SD] HADS-A score, 14.16 [3.17] vs 13.64 [3.20]; adjusted mean difference [AMD], 0.52; 95\% CI, -0.22 to 1.27 ) and depression (mean [SD] HADS-D score, 12.67 [3.27] vs 12.49 [3.34]; AMD, 0.17; 95\% CI, -0.54 to 0.89). One week after treatment completion, the intervention group had significantly lower scores on HADS-A (mean [SD], 7.56 [3.41] vs 10.26 [3.92]; AMD, $-2.77 ; 95 \% \mathrm{CI},-3.72$ to -1.82 ) and HADS-D (mean [SD], 6.49 [3.25] vs 9.45 [3.38]; AMD, -3.02; $95 \% \mathrm{CI},-3.93$ to -2.10 ). After 3 months from the start of treatment, the intervention group had significantly lower scores than the control group on HADS-A (mean [SD], 7.25 [3.63] vs 10.03 [3.87]; AMD, $-2.77 ; 95 \% \mathrm{CI},-3.56$ to -1.98 ) and HADS-D (mean [SD], 6.30 [3.40] vs 9.27 [3.56]; AMD, $-2.98 ; 95 \%$ CI, -3.74 to -2.22 ). The HADS total score also showed similar differences between the 2 groups at 1 week after treatment (mean [SD], 14.02 [6.30] vs 19.71 [6.99]; AMD, -5.83 ; 95\% CI, -7.60 to -4.06 ) and at 3 months' follow-up (mean [SD], 13.55 [6.75] vs 19.29 [7.12]; AMD, -5.75 ; 95\% CI, -7.21 to -4.29 ) (Table 2 and eTable 1 in Supplement 2).

At 3 months after start of the treatment, there were also significant differences in scores of posttraumatic stress (PCL-5 score: AMD, -5.86 ; 95\% CI, -8.53 to -3.19 ), functional impairment (WHODAS 2.0 score: AMD, -4.17 ; 95\% CI, -5.84 to -2.51 ), problems for which the person sought help (PSYCHLOPS score: 


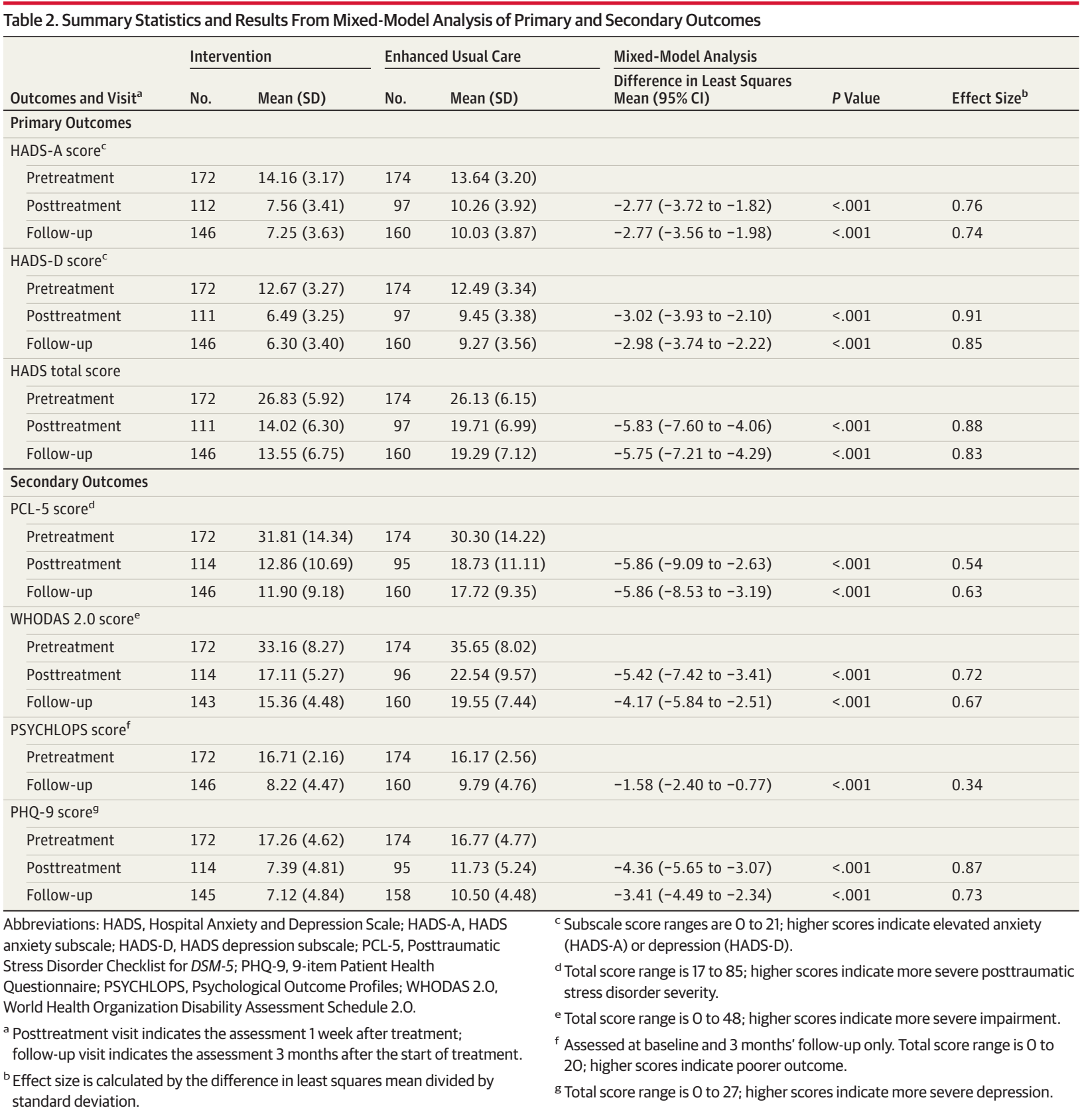

AMD, $-1.58 ; 95 \% \mathrm{CI},-2.40$ to -0.77 ), and symptoms of depressive disorder (PHQ-9 score: AMD, -3.41 ; 95\% CI, -4.49 to -2.34$)$. Post hoc analysis showed that at baseline, $94.2 \%$ of participants in the intervention group and $89.7 \%$ of participants in the control group met the PHQ- 9 criteria for depression. At 3 months after start of the treatment, the rates were $26.9 \%$ and $58.9 \%$, respectively (risk difference, -31.98 ; 95\% CI, -41.03 to -22.94$)$.

The results of the post hoc analysis of primary and secondary outcomes in which the baseline measurement of an outcome was treated as a covariate are presented in Table 3. These results were also significant and consistent with those reported in Table 2 .
The post hoc sensitivity analysis using multiple imputation showed that the estimated treatment effects in terms of primary and secondary end points were insensitive to the missing values (eTable 2 in Supplement 2).

\section{Discussion}

This RCT tested the effectiveness of a brief lay health workeradministered multicomponent intervention in Peshawar, Pakistan, a low-income setting affected by ongoing conflict and insecurity. The outcome evaluation indicated improvements in anxiety, depression, and other secondary outcome 
measures immediately after the 5-week intervention and at 3 months' follow-up. Improvement across all dimensions of anxiety, depression, trauma-related symptoms, and functioning demonstrated the effectiveness of the transdiagnostic feature of the intervention.

A key study strength was that it was successfully conducted in a challenging and insecure real-life setting. Bomb blasts and armed attacks in public places as well as threats and attacks on polio vaccination teams (which operate from primary care centers) were frequent in the area. ${ }^{34}$ The intervention was delivered by the most widely (and in many cases, the only) available human resource for health in such settings: lay workers with no prior mental health care experience. The results show that the lay workers can be successfully trained and supervised to effectively deliver the psychological intervention in primary care settings. The results of this trial demonstrate the feasibility of the taskshifting approach (a task normally performed by a specialist is transferred to a worker with a different or lower level of education and training, or to a person specifically trained to perform a limited task only, without having a formal health education) and are consistent with what our group and others have found in relation to task shifting in mental health care. ${ }^{35-38}$

The study has several limitations. First, we were able to access only about $40 \%$ of the sample at 1-week posttreatment follow-up mainly owing to movement restriction following high security risk to the research team around that time. This was because of attacks on polio vaccination workers and threats of violence to all health care staff. This illustrates the ethical and logistic challenges of undertaking community-based research in conflict settings. ${ }^{39}$ However, the risk of bias due to this limitation is likely to be small: there were similar rates of attrition across both groups of the trial; there was a high response rate at the subsequent 3-month follow-up when the situation was relatively stable; and the use of mixed models in the context of repeated outcome measurement analyses using the random-effects model adjusts for bias induced by missing values. Second, there was an improvement in the control group receiving enhanced usual care. While this could be attributed to regression to the mean, the possible contamination of the control group with elements of intervention cannot be entirely ruled out as the health workers and participants attended the same health care facilities and may have interacted. Third, the duration of sustained benefit or mechanisms for this brief psychological intervention have yet to be determined. Future studies should explore which component mediates effect, how long this lasts, and the feasibility and effect of booster sessions. ${ }^{40}$

The primary outcomes of this trial demonstrated an approximately 3-point difference in both anxiety and depressive scores, which is greater than the minimal clinically important difference calculated for the HADS. ${ }^{21}$ Other studies of nonspecialists, usually community health workers, in lowincome countries have also found similar effects. ${ }^{35}$ A number of factors should be considered when interpreting these findings. The studies were conducted in settings where interven-
Table 3. Summary Results From Post Hoc Mixed-Model Analysis of Primary and Secondary Outcomes, With Baseline Measurement Included as Covariate

\begin{tabular}{|c|c|c|}
\hline $\begin{array}{l}\text { Primary and Secondary } \\
\text { Outcomes and Visit }\end{array}$ & $\begin{array}{l}\text { Difference in Least Squares Mean } \\
\text { for Intervention vs Enhanced } \\
\text { Usual Care }(95 \% \mathrm{Cl})\end{array}$ & $P$ Value \\
\hline \multicolumn{3}{|l|}{ HADS-A score } \\
\hline Pretreatment & $0.29(-0.39$ to 0.97$)$ & .40 \\
\hline Posttreatment & $-2.96(-3.84$ to -2.08$)$ & $<.001$ \\
\hline Follow-up & $-2.99(-3.72$ to -2.27$)$ & $<.001$ \\
\hline \multicolumn{3}{|l|}{ HADS-D score } \\
\hline Pretreatment & $0.10(-0.54$ to 0.74$)$ & .77 \\
\hline Posttreatment & $-3.07(-3.90$ to -2.24$)$ & $<.001$ \\
\hline Follow-up & $-3.08(-3.77$ to -2.40$)$ & $<.001$ \\
\hline \multicolumn{3}{|l|}{ PCL-5 score } \\
\hline Pretreatment & 0.81 ( -1.30 to 2.92$)$ & .45 \\
\hline Posttreatment & $-6.11(-8.83$ to -3.39$)$ & $<.001$ \\
\hline Follow-up & $-6.64(-8.88$ to -4.40$)$ & $<.001$ \\
\hline \multicolumn{3}{|l|}{ WHODAS 2.0 score } \\
\hline Pretreatment & $-1.45(-2.83$ to -0.06$)$ & .04 \\
\hline Posttreatment & $-4.27(-6.06$ to -2.49$)$ & $<.001$ \\
\hline Follow-up & $-3.07(-4.55$ to -1.58$)$ & $<.001$ \\
\hline \multicolumn{3}{|l|}{ PSYCHLOPS ${ }^{\mathrm{b}}$} \\
\hline Pretreatment & $0.19(-0.50$ to 0.89$)$ & .58 \\
\hline Posttreatment & $-2.02(-2.76$ to -1.28$)$ & $<.001$ \\
\hline \multicolumn{3}{|l|}{ PHQ-9 } \\
\hline Pretreatment & $0.26(-0.63$ to 1.16$)$ & .56 \\
\hline Posttreatment & $-4.35(-5.51$ to -3.20$)$ & $<.001$ \\
\hline Follow-up & $-3.69(-4.64$ to -2.73$)$ & $<.001$ \\
\hline
\end{tabular}

Abbreviations: HADS-A, Hospital Anxiety and Depression Scale anxiety subscale; HADS-D, Hospital Anxiety and Depression Scale depression subscale; PCL-5, Posttraumatic Stress Disorder Checklist for DSM-5; PHQ-9, 9-item Patient Health Questionnaire; PSYCHLOPS, Psychological Outcome Profiles; WHODAS 2.0, World Health Organization Disability Assessment Schedule 2.0.

a Posttreatment visit indicates the assessment 1 week after treatment; follow-up visit indicates the assessment 3 months after the start of treatment. ${ }^{\mathrm{b}}$ Assessed at baseline and 3 months' follow-up only.

tions for common mental disorders are practically nonexistent despite the high prevalence of such disorders. It could be hypothesized that in such settings even minimal interventions can have some effect. This was manifested to some extent in the improvement in outcomes found in the control group in this trial. The participants in this trial were primary care attendees identified by a physician for emotional distress and may have been motivated to respond. Moreover, the intervention, while simple to deliver, is based on empirically supported principles. Finally, the lay health workers were recruited from the local population, which means that they could relate well to their clients, and were supervised by more experienced clinicians.

\section{Conclusions}

Among adults impaired by psychological distress in a conflictaffected area, lay health worker administration of a brief 
multicomponent intervention based on established behavioral strategies, compared with enhanced usual care, re- sulted in clinically significant reductions in anxiety and depressive symptoms at 3 months.

\section{ARTICLE INFORMATION}

Published Online: November 12, 2016. doi:10.1001/jama.2016.17165

Author Contributions: Drs Farooq and Wang had full access to all of the data in the study and take responsibility for the integrity of the data and the accuracy of the data analysis.

Concept and design: Rahman, Hamdani, Bryant, Dawson, Azeemi, Chiumento, Sijbrandij, Wang, Farooq, van Ommeren.

Acquisition, analysis, or interpretation of data: Rahman, Hamdani, Awan, Bryant, Dawson, Khan, Akhtar, Nazir, Chiumento, Wang, Farooq, van Ommeren.

Drafting of the manuscript: Rahman, Hamdani, Bryant, Dawson, Sijbrandij, Wang. Critical revision of the manuscript for important intellectual content: Rahman, Hamdani, Awan, Dawson, Khan, Azeemi, Akhtar, Nazir, Chiumento, Sijbrandij, Wang, Farooq, van Ommeren. Statistical analysis: Rahman, Hamdani, Bryant, Akhtar, Nazir, Wang.

Obtained funding: Rahman, Hamdani, Sijbrandij, van Ommeren.

Administrative, technical, or material support: Rahman, Hamdani, Bryant, Dawson, Khan, Azeemi, Chiumento, Sijbrandij, Farooq, van Ommeren.

Study supervision: Rahman, Hamdani, Awan Azeemi, Sijbrandij, Farooq, van Ommeren.

Conflict of Interest Disclosures: All authors have completed and submitted the ICMJE Form for Disclosure of Potential Conflicts of Interest and none were reported.

Funding/Support: This work was supported by Enhanced Learning and Research for Humanitarian Assistance's Research for Health in Humanitarian Crises initiative funded by the UK Department for International Development and the Wellcome Trust.

Role of the Funder/Sponsor: The funders had no role in the design and conduct of the study; collection, management, analysis, and interpretation of the data; preparation, review, or approval of the manuscript; and decision to submit the manuscript for publication.

Disclaimer: The views expressed in this article are those of the authors alone and do not necessarily represent the views, decisions, or policies of the institutions with which the authors are affiliated.

Additional Contributions: We thank the project staff at the Department of Psychiatry, Lady Reading Hospital, Peshawar, Pakistan, for their contributions, the primary health care staff and physicians for their support in the conduct of the study, and the participants and their families for their voluntary participation.

\section{REFERENCES}

1. United Nations Office for the Coordination of Humanitarian Affairs. Global Humanitarian Overview. Geneva, Switzerland: United Nations Office for the Coordination of Humanitarian Affairs; 2016.
2. Steel Z, Chey T, Silove D, Marnane C, Bryant RA, van Ommeren M. Association of torture and other potentially traumatic events with mental health outcomes among populations exposed to mass conflict and displacement: a systematic review and meta-analysis. JAMA. 2009;302(5):537 549.

3. Dua T, Barbui C, Clark N, et al. Evidence-based guidelines for mental, neurological, and substance use disorders in low- and middle-income countries: summary of WHO recommendations. PLOS Med. 2011;8(11):e1001122.

4. Tol WA, Barbui $C$, van Ommeren $M$. Management of acute stress, PTSD, and bereavement: WHO recommendations. JAMA. 2013;310(5):477-478.

5. Saraceno B, van Ommeren M, Batniji R, et al. Barriers to improvement of mental health services in low-income and middle-income countries. Lancet. 2007;370(9593):1164-1174.

6. Kakuma R, Minas H, van Ginneken N, et al. Human resources for mental health care: current situation and strategies for action. Lancet. 2011;378 (9803):1654-1663.

7. Collins PY, Patel V, Joestl SS, et al; Scientific Advisory Board and the Executive Committee of the Grand Challenges on Global Mental Health. Grand challenges in global mental health. Nature. 2011;475(7354):27-30.

8. Dawson KS, Bryant RA, Harper M, et al. Problem Management Plus (PM+): a WHO transdiagnostic psychological intervention for common mental health problems. World Psychiatry. 2015;14(3):354-357.

9. Bernal G, Sáez-Santiago E. Culturally centered psychosocial interventions. J Community Psychol. 2006;34(2):121-132. doi:10.1002/jcop.20096

10. Rahman A, Riaz N, Dawson KS, et al. Problem Management Plus (PM+): pilot trial of a WHO transdiagnostic psychological intervention in conflict-affected Pakistan. World Psychiatry. 2016, 15(2):182-183.

11. Sijbrandij M, Farooq $S$, Bryant RA, et al. Problem Management Plus (PM+) for common mental disorders in a humanitarian setting in Pakistan; study protocol for a randomised controlled trial (RCT). BMC Psychiatry. 2015;15:232.

12. Goldberg DP. A User's Guide to the General Health Questionnaire. Windsor, England: NFER-Nelson; 1988.

13. Minhas F, Mubbashar MH. Validation of General Health Questionnaire (GHQ-12) in primary care settings of Pakistan. J Coll Physicians Surg Pak. 1996:6:133-136.

14. World Health Organization. Measuring Health and Disability: Manual for WHO Disability Assessment Schedule WHODAS 2.0. Geneva, Switzerland: World Health Organization; 2010.

15. World Health Organization. Problem Management Plus (PM+): Individual Psychological Help for Adults Impaired by Distress in Communities Exposed to Adversity (Generic Field-Trial Version 1.0) Geneva, Switzerland: World Health Organization;
2016. http://www.who.int/mental health /emergencies/problem_management_plus/en/. Accessed November 1, 2016

16. Murray LK, Dorsey S, Bolton $P$, et al. Building capacity in mental health interventions in low resource countries: an apprenticeship model for training local providers. Int J Ment Health Syst. 2011; 5(1):30

17. Karim S, Saeed K, Rana MH, Mubbashar MH, Jenkins R. Pakistan mental health country profile. Int Rev Psychiatry. 2004;16(1-2):83-92.

18. Zigmond AS, Snaith RP. The Hospital Anxiety and Depression Scale. Acta Psychiatr Scand. 1983; 67(6):361-370.

19. Mumford DB, Tareen IA, Bajwa MA, Bhatti MR Karim R. The translation and evaluation of an Urdu version of the Hospital Anxiety and Depression Scale. Acta Psychiatr Scand. 1991;83(2): 81-85.

20. Herrmann C. International experiences with the Hospital Anxiety and Depression Scale-a review of validation data and clinical results. J Psychosom Res. 1997;42(1):17-41.

21. Puhan MA, Frey $M$, Büchi S, Schünemann HJ. The minimal important difference of the Hospital Anxiety and Depression Scale in patients with chronic obstructive pulmonary disease. Health Qual Life Outcomes. 2008;6:46.

22. Weathers FW, Litz BT, Keane TM, Palmieri PA, Marx BP, Schnurr PP. The PTSD Checklist for DSM-5 (PCL-5). http://www.ptsd.va.gov/professional /assessment/adult-sr/ptsd-checklist.asp. Published 2013. Accessed November 1, 2016.

23. Blanchard EB, Jones-Alexander J, Buckley TC, Forneris CA. Psychometric properties of the PTSD Checklist (PCL). Behav Res Ther. 1996;34(8):669673.

24. Khalily MT, Gul S, Mushtaq R, Jahangir SF. To examine delayed PTSD symptomatology over time among trauma survivors in Pakistan. Online J Couns Educ. 2012;1(1):1-11.

25. Ustün TB, Chatterji S, Kostanjsek N, et al; WHO/NIH Joint Project. Developing the World Health Organization Disability Assessment Schedule 2.0. Bull World Health Organ. 2010;88(11): 815-823.

26. Ashworth M, Shepherd M, Christey J, et al. A client-generated psychometric instrument: the development of "PSYCHLOPS." Couns Psychother Res. 2004;4(2):27-31. doi:10.1080 /14733140412331383913

27. Ashworth M, Robinson SI, Godfrey E, et al. Measuring mental health outcomes in primary care: the psychometric properties of a new patient-generated outcome measure, 'PSYCHLOPS' ('psychological outcome profiles'). Prim Care Ment Health. 2005;3(4):261-270.

28. Kroenke K, Spitzer RL, Williams JB. The PHQ-9. validity of a brief depression severity measure. J Gen Intern Med. 2001;16(9):606-613.

29. Husain N, Gater R, Tomenson B, Creed F. Comparison of the personal health questionnaire and the self reporting questionnaire in rural Pakistan. J Pak Med Assoc. 2006;56(8):366-370. 
30. Ahmer S, Faruqui RA, Aijaz A. Psychiatric rating scales in Urdu: a systematic review. BMC Psychiatry. 2007;7:59.

31. Rahman A, lqbal Z, Harrington R. Life events, social support and depression in childbirth: perspectives from a rural community in the developing world. Psychol Med. 2003;33(7):1161-1167.

32. Patel V, Weiss HA, Chowdhary N, et al. Effectiveness of an intervention led by lay health counsellors for depressive and anxiety disorders in primary care in Goa, India (MANAS): a cluster randomised controlled trial. Lancet. 2010;376 (9758):2086-2095

33. Raudenbush SW. Comparing persona trajectories and drawing causal inferences from longitudinal data. Annu Rev Psychol. 2001;52:501525.
34. Mohanty TR. Pakistan: KP: rising graph of violence. South Asia Intell Rev. 2014;12(33). http://www.satp.org/satporgtp/sair/Archives/sair12 /12_33.htm. Accessed November 1, 2016.

35. van Ginneken N, Tharyan P, Lewin S, et al. Non-specialist health worker interventions for the care of mental, neurological and substance-abuse disorders in low- and middle-income countries. Cochrane Database Syst Rev. 2013;11(11):CD009149.

36. Bass JK, Annan J, Mclvor Murray S, et al. Controlled trial of psychotherapy for Congolese survivors of sexual violence. N Engl J Med. 2013; 368(23):2182-2191.

37. Bolton P, Lee C, Haroz EE, et al. A transdiagnostic community-based mental health treatment for comorbid disorders: development and outcomes of a randomized controlled trial among Burmese refugees in Thailand. PLoS Med. 2014;11(11):e1001757.

38. Rahman A, Malik A, Sikander S, Roberts C, Creed F. Cognitive behaviour therapy-based intervention by community health workers for mothers with depression and their infants in rural Pakistan: a cluster-randomised controlled trial. Lancet. 2008;372(9642):902-909.

39. Chiumento A, Khan MN, Rahman A, Frith L. Managing ethical challenges to mental health research in post-conflict settings. Dev World Bioeth. 2016;16(1):15-28.

40. Natsuaki MN. Intervention effect fades over time: when, how, and for whom? Lancet Psychiatry. 2015:2(7):573-574. 